\title{
Is early diagnosis of myofascial pain syndrome possible with the detection of latent trigger points by shear wave elastography?
}

\author{
Ersen Ertekin ${ }^{1 A, B, C, D, E, F}$, Zehra S. Kasar ${ }^{2 A, B, E, F}$, Figen Tunali Turkdogan ${ }^{1 A, D, E}$ \\ 'Department of Radiology, Faculty of Medicine, Aydın Adnan Menderes University, Aydın, Turkey \\ 2Department of Physical Therapy and Rehabilitation, Faculty of Medicine, Aydın Adnan Menderes University, Aydın, Turkey
}

\begin{abstract}
Purpose: The aim of the study was to investigate the contribution of shear wave elastography to the diagnosis of myofascial pain syndrome (MPS) of the upper part of the trapezius.

Material and methods: Ethical committee approval was obtained for the study. Thirty volunteer women with trigger points in the upper part of the trapezius muscle and 30 healthy women with a similar age distribution were included in the study. The patient group performed a self-stretching exercise program for 4 weeks. No intervention was applied to the control group. Muscle stiffness values of both groups were evaluated with shear wave elastography (SWE), and pain levels of all volunteers were evaluated by the Visual Analogue Scale at the beginning and the end of the study. The statistical analyses were performed using SPSS version 18.0.
\end{abstract}

Results: There was a significant decrease after the treatment in terms of upper trapezius muscle stiffness and the pain levels in the patient group $(p<0.001$ and $p<0.001)$. In the patient group, there was a moderate correlation between the decrease in the pain level and the reductions in muscle stiffness $(r=0.595)$. In control group, there was no significant difference in terms of both muscle stiffness and pain levels before and after treatment $(p>0.05)$.

Conclusions: SWE is a reliable method for detecting latent trigger points in MPS, and it can be used for evaluating the response to treatment.

Key words: myofascial pain syndrome, latent trigger point, shear wave elastography.

\section{Introduction}

Myofascial pain syndrome (MPS), previously called muscular rheumatism and non-joint rheumatism, is a soft tissue pain syndrome characterized by local and/or directed pain originating from trigger points (TrPs) [1]. In contrast to fibromyalgia, which is a generalized pain syndrome, MPS is limited to a specific body area. The most commonly affected muscle in the neck-shoulder girdle in MPS is the trapezius muscle [2]. The aetiology of MPS is not yet fully understood. The characteristic finding of MPS is the painful TrPs due to the sensitivity of nodules within the taut bands of the skeletal muscle [1-3]. Although there have not been generally accepted definitive diagnostic criteria, the diagnosis of MPS is based on the detection of these TrPs [4].

TrPs causing MPS can be active or latent. If a TrP spontaneously triggers pain, it is called 'Active TrP'. A Latent TrP has all the features of a TrP but is clinically silent. Latent TrPs can be transformed into active form and cause clinical symptoms [5]. Therefore, early diagnosis and treatment before clinical activation is very important.

The pathophysiology of MPs is not fully known. According to the motor phenomenon, increased acetylcholine $(\mathrm{ACh})$ in the motor end plate leads to permanent depolarization of the muscle fibre membrane, resulting in excessive

Correspondence address:

Asst. Prof. Dr. Ersen Ertekin, MD, Department of Radiology, Faculty of Medicine, Aydın Adnan Menderes University, Efeler/Aydın, Turkey,

e-mail: drersen@hotmail.com

Authors' contribution:

A Study design · B Data collection · C Statistical analysis · D Data interpretation · E Manuscript preparation · F Literature search · G Funds collection 
muscle contraction. Hypoperfusion/ischaemia due to excessive muscle contraction lead to increased mediators and acidification triggering a vicious cycle. This vicious cycle causes excessive stimulation of nociceptors and hence pain $[1,3,5]$. The main objective of treatment is breaking this cycle. Although some alternative therapies such as dry needling, analgesic injection, non-steroidal anti-inflammatory drugs (NSAID), acupuncture, or Botulinum toxin A are used in treatment, stretching exercises are the basis of MPS treatment [6,7].

The resting stiffness of the muscles involved in TrPs is high $[2,4]$. The stiffness of these muscles can be determined by US elastography. US elastography has been widely used in many tissues and organs, including the breast, thyroid, liver, and muscles/tendons. Although there are several techniques, the most reliable elastography method is shear wave elastography (SWE) $[8,9]$. Due to the ability of detecting micro-environmental changes earlier than ultrasound and providing quantitative information about tissue stiffness, the utilization of SWE has been increasing day by day [8-11].

Considering this information, we investigated whether SWE can detect latent TrPs as well as active TrPs in MPS and whether it may contribute to the diagnosis of MPS in the upper part of the trapezium muscle. We also investigated the usability of SWE for evaluating the efficacy of a stretching exercise program.

\section{Material and methods}

This study was approved by the Ethical Committee for Non-Interventional Clinical Trials within our institution. Before the study, a signed voluntary consent form was obtained from all participants.

\section{Patient selection}

The volunteers' trapezius muscles were evaluated with physical examination by a physiotherapist with more than 20 years of experience. The diagnostic criteria determined by Travel and Simons [8] were used for MPS, the diagnosis of which requires 5 major criteria and at least 1 minor criterion. The major criteria include the following: (I) localised spontaneous pain; (II) spontaneous pain or altered sensations in the expected referred area for a given $\operatorname{TrP}$; (III) a palpable taut band in an accessible muscle; (IV) exquisite, localised tenderness in a precise point along the taut band; (V) a certain degree of reduced range of movement when measured. Minor criteria include the following: (I) reproduction of spontaneously perceived pain and altered sensations by pressure on the TrP; (II) elicitation of a local twitch response (LTR) of muscle fibres by transverse 'snapping' palpation or by needle insertion into the TrP; (III) pain relieved by muscle stretching or injection of the $\operatorname{TrP}$.
Thirty volunteers who had taut band and $\operatorname{Tr} P$ were included in the study. Patients with cervical disc herniation, radiculopathy or myelopathy, tumoural or infectious processes, or psychiatric or systemic disease were excluded from the study. Thirty healthy volunteers with similar age distribution to the MPS group, who had no active pain and had no taut band or $\operatorname{TrP}$ in their physical examination, were determined as the control group.

\section{Patient evaluation}

For the detection of taut bands and TrPs, all volunteers were physically examined at the beginning of the study. During physical examination, the pain levels of all volunteers were evaluated using the visual analogue scale (VAS). The participants were asked to score their pain levels from 0 to 10 .

Then, the volunteers in the MPS group were included in the stretching exercise program for 4 weeks for the upper part of the trapezius. The volunteers performed the stretching and posture exercises on their own 3 times a day and performed each movement 10 times. For providing effective treatment, the volunteers were trained by performing their first exercises under the supervision of a physiotherapist. The volunteers in the control group did not perform any exercise program.

At the end of the exercise program, the study group was evaluated with a physical examination by the same physiotherapist in terms of taut bands, TrPs, and pain levels. Similarly, the control group was re-evaluated 4 weeks after the first examination and pain levels were obtained.

\section{Ultrasound elastography examination}

The elastography measurements of all volunteers were performed by 2 radiologists who were unaware of each other and the group of patients, and who had 13 and 14 years of US experience, respectively. A maximum of 5 minutes elapsed between the measurements of the radiologists.

All SWE measurements were performed with a 9-12 MHz linear probe on a Samsung RS 80 (Gyeonggi-do, Republic of Korea) ultrasound device. The upper parts of the trapezius muscle of all participants were evaluated twice with a 4-week interval. For the standardization of the test, measurements were performed on the middle of an imaginary line between the processus spinosus of the $7^{\text {th }}$ cervical vertebra and the acromion. During the measurements, the volunteers were in a sitting position with their hands on their knees in full resting position and their head was upright and in the midline. The probe was placed in a position parallel to the muscle fibres. For each volunteer, at least 10 measurements were performed, and the average of these measurements was recorded. The elasticity is shown in kilopascals $(\mathrm{kPa})$. 
Table 1. Shear wave elastography measurements of the upper part of the trapezius muscles and statistical analysis for the groups

\begin{tabular}{|c|c|c|c|}
\hline & MPS group $(n=30)$ & Control group $(n=30)$ & $p$-value \\
\hline SWE ${ }^{1}$ first $(\mathrm{kPa})$, mean $\pm \mathrm{SD}$ & $60.3 \pm 15.8$ & $41.7 \pm 9.6$ & $<0.001^{*}$ \\
\hline $\mathrm{SWE}^{2}$ first $(\mathrm{kPa})$ mean $\pm \mathrm{SD}$ & $60.7 \pm 17.7$ & $41.7 \pm 10.1$ & $<0.001^{*}$ \\
\hline$S W E^{1}$ last $(\mathrm{kPa})$, mean $\pm \mathrm{SD}$ & $44.2 \pm 12.0$ & $38.5 \pm 8.9$ & 0.042 \\
\hline SWE ${ }^{2}$ last $(\mathrm{kPa})$, mean $\pm \mathrm{SD}$ & $44.4 \pm 13.9$ & $39.4 \pm 9.3$ & 0.111 \\
\hline SWE ${ }^{1}$ differ (kPa), mean (min/max) & $16.1(+3.5 /+35.1)$ & $3.2(-4.2 /+13.6)$ & $<0.001^{*}$ \\
\hline SWE $E^{2}$ differ (kPa), mean (min/max) & $16.3(-1.5 /+42.1)$ & $2.3(-5.9 /+10.2)$ & $<0.001^{*}$ \\
\hline
\end{tabular}

${ }^{1}$ First radiologist. ${ }^{2 S}$ Second radiologist. MPS - myofascial pain syndrome, kPa - kilopascal, differ - difference. * Statistically significant

\section{Statistical analysis}

For statistical analysis of data the $t$-test, Pearson's correlation test for parametric data, and Spearman's correlation test for nonparametric data were performed in SPSS 18.0 program. SWE measurements of 2 radiologists were evaluated for inter-observer reliability.

\section{Results}

Sixty volunteer women with a mean age of $20.6 \pm 1.9$ years (mean \pm SD) participated in the study. The mean age was $20.3 \pm 1.7$ years (mean \pm SD) and $20.9 \pm 2.2$ years (mean $\pm \mathrm{SD}$ ) in the exercise and control groups, respectively. There was no significant difference between the 2 groups in terms of age $(p=0.190)$.

The mean pre-treatment SWE measurements of the volunteers were $51.0 \pm 16.0$ (mean \pm SD) for the first radiologist and $51.2 \pm 17.2$ (mean $\pm \mathrm{SD}$ ) for the second radiologist. Overall mean of the post-treatment SWE measurements was $41.4 \pm 10.9$ (mean \pm SD) for the first radiologist and $41.9 \pm 12.0$ (mean \pm SD) for the second radiologist. The overall mean differences between SWE values before and after treatment were $9.7(\min / \max :-4.2 /+35.1)$ for the first radiologist and $9.3(\mathrm{~min} / \mathrm{max}:-5.9 /+42.1)$ for the second radiologist. The distribution of the SWE data according to the groups is summarized in Table 1.

There was a high correlation between the SWE measurements of the 2 radiologists before and after treatment ( $r=0.97, r=0.93$, respectively). Figure 1 shows the compatibility between the SWE measurements of both radiologists.

The difference between SWE measurements of both radiologists before and after treatment was statistically significant $(p<0.001)$ in the exercise group whereas no significant difference was observed in the control group $(p>0.05)$. Changes in SWE measurements of a case with latent $\mathrm{TrP}$ and a volunteer in the control group are shown in Figures 2 and 3, respectively.

The decrease in VAS score after treatment in the exercise group was statistically significant when compared to the control group $(p<0.001)$.

In the exercise group, there was a moderate positive correlation between the decrease in VAS score and the

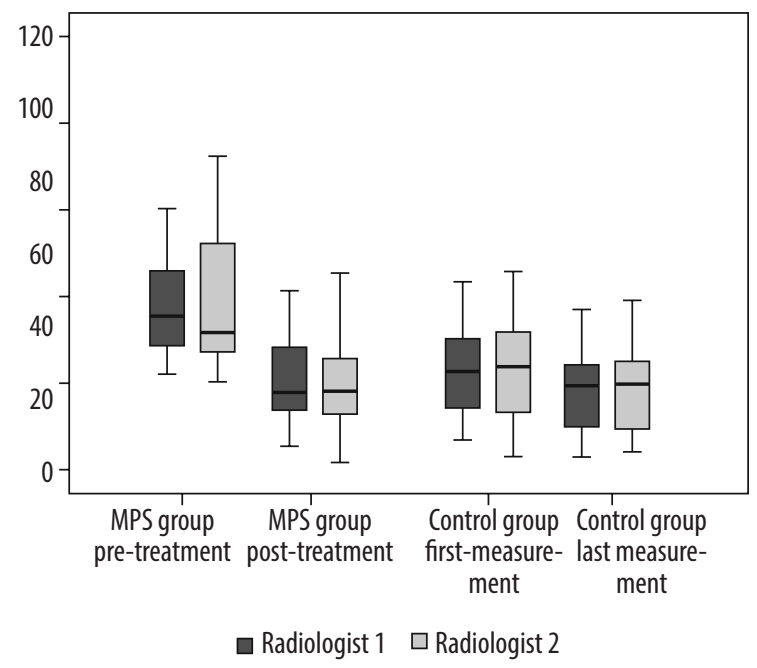

Figure 1. Box-plot graphic of the first and last shear wave elastography (SWE) measurements of 2 radiologists in the latent trigger point and the control groups. It shows the compatibility of SWE measurements of the 2 radiologists

difference between SWE values before and after treatment ( $r=0.570, r=595$, respectively).

\section{Discussion}

In this study, we evaluated the trapezius muscle stiffness with SWE in volunteers with latent TrP, and the muscle stiffness of the participants with myofascial TrP was significantly higher than that of the control group. Similarly, the pain levels in the MPS group were higher than those of the control group. In the MPS group, which performed a passive stretching exercise program for 4 weeks, the decrease of the stiffness of trapezius muscle was demonstrated by SWE with high accuracy.

MPS is a syndrome characterized by localized and/or directed pain caused by TrPs, which are limited to a specific body area [1]. The prevalence of MPS, which is one of the most important causes of functional impairment in daily life, is not exactly known due to the absence of generally accepted diagnostic criteria [1,2]. MPS is mostly seen in the neck-shoulder region, and the most commonly involved muscle is the upper part of the trapezius $[2,3]$. Although the aetiology is not known, factors such as muscle injuries overuse of muscles, repetitive move- 


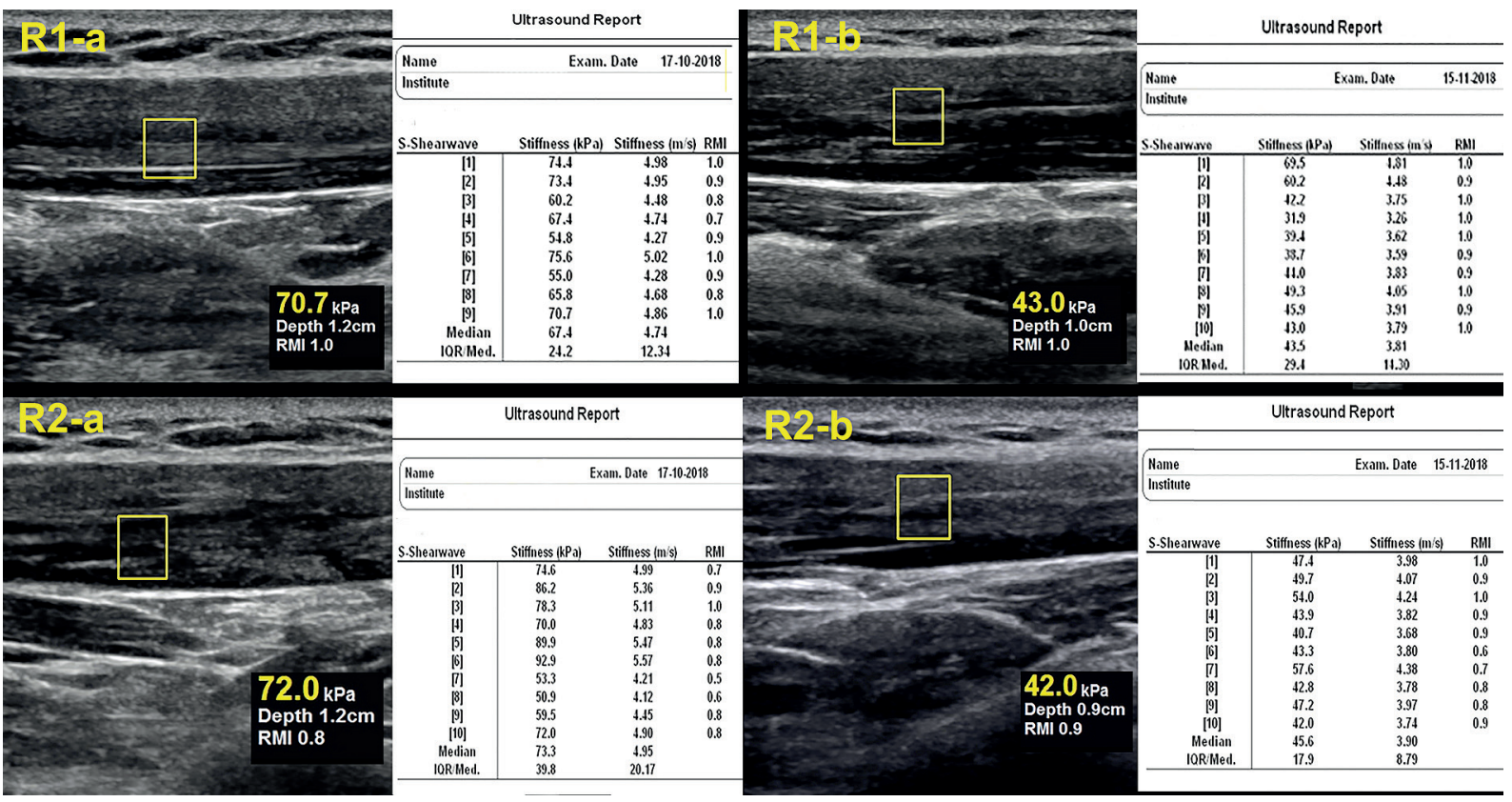

Figure 2. 22-year-old female case with latent trigger point. Shear wave elastography measurements of both radiologists (R1 and R2) showed a significant decrease in (a) pre-treatment (b) measurements



Figure 3. Shear wave elastography (SWE) measurements of 21-year-old female volunteers in the control group. No significant change was observed in the first (a) and last (b) SWE measurements of both radiologists ( $R 1$ and R2)

ments, poor working conditions, inactivity, anxiety, and depression may cause development of myofascial pain or aggravation of the clinical picture $[2,3,12]$.

The diagnosis of MPS is difficult because of the lack of generally accepted diagnostic criteria. The most commonly used findings for diagnosis are the presence of taut bands, local or directed pain occurring spontaneously or by palpation in a specific region, twitch response with palpation of the taut band, observation of the jump sign, limitation of movement in the affected joint, and symptom resolution with applied local anaesthetic treatments $[4,5]$.
The diagnosis is largely based on the detection of TrPs in physical examination. During the palpation, localized or regional extreme pain occurs on TrPs. TrPs causing MPS may be active or latent. Latent TrPs are clinically silent, while spontaneous pain can be triggered in active TrPs. However, the latent TrPs may turn into an active form in subsequent periods and may cause clinical findings [1-5]. For this reason, early diagnosis and treatment without clinical picture is very important. It is known that resting stiffness of muscles is high in MPS patients $[2,4]$. Although they are clinically silent, the Latent TrPs can be 
expected to have high muscle stiffness because they have all the features of active TrPs.

Sonoelastography can detect increased resting strain in MPS, as well as detecting microenvironmental changes in pathological tissues earlier than ultrasound [10-13]. In our literature review, we found only 2 studies with sonoelastography in patients with Latent TrPs $[14,15]$. In their study, Calvo-Lobo et al. [14] compared active and latent TrPs with the control group in the lumbar erector spina muscles by sonoelastography, and they found high muscle stiffness in both TrP types according to the control group, while active and latent TrPs did not show any significant difference. Grabowski et al. [15] evaluated the infraspinatus muscles of at least one Latent $\operatorname{Tr} P$ by sonoelastography. They noted that the stiffness of the affected muscle and TrPs was not significantly different; however, both the muscle and the TrPs were stiffer than in the control group. We did not find any studies that evaluated latent TrPs in the upper trapezius muscle by sonoelastography. Our study will make a significant contribution to the literature on this subject.

Sonoelastography technique can be divided into 2 main groups: strain elastography (SE) and shear wave elastography (SWE). In SE, which is based on the tissue texture change against an applied external force, the resistance of the tissue to deformation can be considered semi-quantitatively according to a reference tissue. In SWE, by measuring the deviations in the velocities of internal shear waves, a quantitative evaluation can be made $[8,13]$. Therefore, SE is a highly user-dependent technique, whereas SWE is user-independent. In the current study, SWE measurements were performed by 2 radiologists who were unaware of the diagnosis and each other, and interobserver reliability for these 2 radiologists was quite high. This supports the reliability of the used method.

In the present study, we found that the trapezius muscle stiffness of the volunteers who had latent TrPs was significantly higher than that that of the control group. This finding is consistent with the increased stiffness of the trapezius muscle in patients with neck pain in the literature [14,16,17]. Taş et al. [16] compared 35 patients with at least 1 year of neck pain and 35 asymptomatic healthy subjects, and they found that the upper trapezius, levator scapula and sternocleidomastoid (SCM) muscle stiffness were significantly higher in the patient group. In their study, Jafari et al. [17] had SWE measurements with and without application of stress to the SCM muscle in 29 patients, and showed that the muscle stiffness increased under stress.

Although the pathophysiology of MAS is largely unknown, the motor phenomenon is generally accepted in the mechanism of contractions. According to the motor phenomenon, it is assumed that increased acetylcholine (ACh) release leads to permanent depolarization of the muscle fibre membrane in the motor end plate, resulting in continuous sarcomere shortening and contractions
$[3,4]$. Excess-contracted muscle fibres (taut bands) can lead to hypoperfusion and areas of ischaemia due to their high energy consumption. This vicious cycle sensitizes nociceptors and causes pain $[3,12,18]$. As the contractions in the taut bands increase, it may cause to the increased pain due to the vicious cycle. However, in the current study, we did not find a correlation between the pain severity and muscle stiffness, similarly to Taş et al. [16]. Unlike Taş et al., we determined the change in pain severity by repeating the VAS scoring after treatment in our study, and we found a high correlation between the change in SWE and the change in pain scale. This finding indicates that SWE can be used to evaluate response to treatment in addition to the diagnosis of MPS.

The main principle in the treatment of MPS is to break the vicious cycle of pain-spasm. Although some methods such as dry needling, solution injections to TrPs, non-steroidal anti-inflammatory drugs, acupuncture, therapeutic ultrasound, and Botulinum A toxin injection [19-22] can be used for the treatment, exercise is the easiest, cheapest, and most effective treatment option [23-25]. Stretching exercises are the basis of exercise therapy in myofascial pain. More effective results have been reported in individuals with an early stage of TrPs [3]. In the present study, we used a self-administered exercise program because we focused mainly on the use of SWE for evaluating the response to treatment and because the participants had no symptoms with Latent TrPs. We found a significant decrease in the pain scores before and after treatment in the MPS group, and this indicates that SWE can be used to evaluate response to treatment. The effectiveness of other treatment modalities can also be evaluated objectively with SWE.

\section{Limitations}

The inability to compare the active $\operatorname{TrP}$ and Latent $\operatorname{TrPs}$ due to the patient group consisting of clinically silent cases may be considered as a limitation. However, we specifically planned to study the clinically silent group and to investigate the diagnostic capacity and treatment evidence of these individuals. The second limitation is the inability to evaluate changes in muscle tonus according to age due to the relatively young study sample. However, we think that we had a healthier outcome of SWE by minimizing the effect of the patients' ages. Another limitation is utilization of only an exercise programme for treatment. But choosing other treatment methods for clinically silent volunteers may have caused ethical problems. Therefore, we decided to practice only the stretching exercise programme.

\section{Conclusions}

SWE, which is an objective and reliable technique with quantitative data, can contribute to the diagnosis of MPS 
and detect latent TrPs that are clinically silent, and it allows treatment to be applied before clinical findings occur. It can also be used as a reliable method for evaluating treatment efficacy in patients with MPS.

\section{Conflict of interest}

The authors report no conflict of interest.

\section{References}

1. Cummings M, Baldry P. Regional myofascial pain: diagnosis and management. Best Pract Res Clin Rheumatol 2007; 21: 367-387.

2. Borg-Stein J, Simons DG. Focused review: myofascial pain. Arch Phys Med Rehabil 2002; 83: 40-49.

3. Giamberardino MA, Affaitati G, Fabrizio A, Costantini R. Myofascial pain syndromes and their evaluation. Best Pract Res Clin Rheumatol 2011; 25: 185-198.

4. Nicol AL, Crooks M, Hsu ES, Ferrante FM. Mysofascial pain syndrome. In: Benzon HT, Raja SN, Liu SS, Fishman SM, Cohen SP (eds.). Essentials of Pain Medicine. $4^{\text {th }}$ ed. Elsevier, Philadelphia 2018. p. 207-12.e1.

5. Simons, DG. Travell, JG. Simons PT. Trapezius muscle. In: Johnson EP, Napora LS, Myers JS (eds.). Travell and Simons' Myofascial Pain and Dysfunction: the Trigger Point Manual. Vol I. Upper half of body. $2^{\text {nd }}$ ed. Williams and Wilkins, Baltimore 1999. p. 278-307.

6. Martin BI, Deyo RA, Mirza SK, et al. Expenditures and health status among adults with back and neck problems. JAMA 2008; 299 : 656-664.

7. Ichihashi N, Umegaki H, Ikezoe T, et al. The effects of a 4-week static stretching programme on the individual muscles comprising the hamstrings. J Sports Sci 2016; 34: 2155-2159.

8. Sigrist RMS, Liau J, Kaffas AE, et al. Ultrasound elastography: review of techniques and clinical applications. Theranostics 2017; 7: 1303-1329.

9. Yuan S, Magarik M, Lex AM, Fleischer AC. Clinical applications of sonoelastography. Expert Rev Med Devices 2016; 13: 1107-1117.

10. Schmalzl J, Fenwick A, Boehm D, Gilbert F. The application of ultrasound elastography in the shoulder. J Shoulder Elbow Surg 2017; 26: 2236-2246.

11. Taljanovic MS, Gimber LH, Becker GW, et al. Shear-wave elastography: basic physics and musculoskeletal applications. Radiographics 2017; 37: 855-870.

12. Heizelmann A, Tasdemir S, Schmidberger J, et al. Measurements of the trapezius and erector spinae muscles using virtual touch imaging quantification ultrasound-elastography: a cross section study. BMC Musculoskelet Disord 2017; 18: 370.

13. Gennisson JL, Deffieux T, Fink M, Tanter M. Ultrasound elastography: principles and techniques. Diagn Interv Imaging 2013; 94: 487-495.

14. Calvo-Lobo C, Diez-Vega I, Martínez-Pascual B, et al. Tensiomyography, sonoelastography, and mechanosensitivity differences be- tween active, latent, and control low back myofascial trigger points: a cross-sectional study. Medicine (Baltimore) 2017; 96: e6287.

15. Grabowski PJ, Slane LC, Thelen DG, et al. Evidence of generalized muscle stiffness in the presence of latent trigger points within infraspinatus. Arch Phys Med Rehabil 2018; 99: 2257-2262.

16. Taş S, Korkusuz F, Erden Z. Neck muscle stiffness in participants with and without chronic neck pain: a shear-wave elastography study. J Manipulative Physiol Ther 2018; 41: 580-588.

17. Jafari M, Bahrpeyma F, Mokhtari-Dizaji M, Nasiri A. Novel method to measure active myofascial trigger point stiffness using ultrasound imaging. J Bodyw Mov Ther 2018; 22: 374-378.

18. Money S. Pathophysiology of trigger points in myofascial pain syndrome. J Pain Palliat Care Pharmacother 2017; 31: 158-159.

19. Liu L, Huang QM, Liu QG, et al. Effectiveness of dry needling for myofascial trigger points associated with neck and shoulder pain: a systematic review and meta-analysis. Arch Phys Med Rehabil 2015; 96: 944-955.

20. Nouged E, Dajani J, Ku B, et al. Local anesthetic injections for the short-term treatment of head and neck myofascial pain syndrome: a systematic review with meta-analysis. J Oral Facial Pain Headache 2019; 33: 183-198.

21. Khalifeh M, Mehta K, Varguise N, et al. Botulinum toxin type A for the treatment of head and neck chronic myofascial pain syndrome: a systematic review and meta-analysis. J Am Dent Assoc 2016; 147: 959-73.e1

22. Wang R, Li X, Zhou S, et al. Manual acupuncture for myofascial pain syndrome: a systematic review and meta-analysis. Acupunct Med 2017; 35: 241-250.

23. Kalichman L, Ben David C. Effect of self-myofascial release on myofascial pain, muscle flexibility, and strength: a narrative review. J Bodyw Mov Ther 2017; 21: 446-451.

24. Oliveira-Campelo NM, de Melo CA, Alburquerque-Sendín F, Machado JP. Short- and medium-term effects of manual therapy on cervical active range of motion and pressure pain sensitivity in latent myofascial pain of the upper trapezius muscle: a randomized controlled trial. J Manipulative Physiol Ther 2013; 36: 300-309.

25. Ahmed S, Khattab S, Haddad C, et al. Effect of aerobic exercise in the treatment of myofascial pain: a systematic review. J Exerc Rehabil 2018; 14: 902-910. 